\title{
A Cone-Shaped Phantom for Assessment of Small Animal PET Scatter Fraction and Count Rate Performance
}

\author{
Rameshwar Prasad, ${ }^{1}$ Habib Zaidi ${ }^{1,2,3}$ \\ ${ }^{1}$ Division of Nuclear Medicine and Molecular Imaging, Geneva University Hospital, 1211 Geneva, Switzerland \\ ${ }^{2}$ Geneva Neuroscience Center, Geneva University, 1211 Geneva, Switzerland \\ ${ }^{3}$ Department of Nuclear Medicine and Molecular Imaging, University Medical Center Groningen, University of Groningen, 9700 \\ Groningen, the Netherlands
}

\begin{abstract}
Purpose: Positron emission tomography (PET) image quality deteriorates as the object size increases owing to increased detection of scattered and random events. The characterization of the scatter component in small animal PET imaging has received little attention owing to the small scatter fraction (SF) when imaging rodents. The purpose of this study is first to design and fabricate a cone-shaped phantom which can be used for measurement of object size-dependent SF and noise equivalent count rates (NECR), and second, to assess these parameters for two small animal PET scanners as function of radial offset, object size and lower energy threshold (LET).

Methods: The X-PET'TM and LabPET-8 ${ }^{\mathrm{TM}}$ scanners were modeled as realistically as possible using GATE Monte Carlo simulation platform. The simulation models were validated against experimental measurements in terms of sensitivity, SF and NECR. The dedicated phantom was fabricated in-house using high-density polyethylene. The optimized dimensions of the coneshaped phantom are $158 \mathrm{~mm}$ (length), $20 \mathrm{~mm}$ (minimum diameter), $70 \mathrm{~mm}$ (maximum diameter) and taper angle of $9^{\circ}$.

Results: The relative difference between simulated and experimental results for the LabPET-8 $8^{\mathrm{TM}}$ scanner varied between $0.7 \%$ and $10 \%$ except for a few results where it was below $16 \%$. Depending on the radial offset from the center of the central axial field-ofview (3-6 cm diameter), the SF for the cone-shaped phantom varied from $26.3 \%$ to $18.2 \%$, 18.6 to $13.1 \%$ and 10.1 to $7.6 \%$ for the X-PET ${ }^{\mathrm{TM}}$, whereas it varied from $34.4 \%$ to $26.9 \%$, 19.1 to $17.0 \%$ and 9.1 to $7.3 \%$ for the LabPET-8 ${ }^{\text {TM }}$, for LETs of 250,350 and $425 \mathrm{keV}$, respectively. The SF increases as the radial offset decreases, LET decreases and object size increases. The SF is higher for the LabPET-8 ${ }^{\mathrm{TM}}$ compared with the X-PET ${ }^{\mathrm{TM}}$ scanner. The NECR increases as the radial offset increases and object size decreases. The maximum NECR was obtained at a LET of $350 \mathrm{keV}$ for the LabPET-8 ${ }^{\mathrm{TM}}$ and $250 \mathrm{keV}$ for the X-PET'M . High correlation coefficients for SF and NECR were observed between the cone-shaped phantom and an equivalent volume cylindrical phantom for the three considered axial fields of view.

Conclusions: A single cone-shaped phantom enables the assessment of the impact of three factors, namely radial offset, LET and object size on PET SF and count rate estimates. This phantom is more realistic owing to the non-uniform shape of rodents' bodies compared to
\end{abstract}

Correspondence to: Habib Zaidi; e-mail: habib.zaidi@hcuge.ch 
cylindrical uniform phantoms and seems to be well suited for evaluation of object sizedependent SF and NECR.

Key words: PET, Small animals, Scatter, Count rate, Monte Carlo simulation

\section{Introduction}

$\mathrm{P}$ ositron emission tomography (PET), a non-invasive molecular imaging modality, is being widely used for qualitative characterization and quantification of biochemical processes in vivo. PET scanners dedicated to small-animal imaging provide the high spatial resolution and high sensitivity required for in vivo molecular imaging [1]. This steered the development of various innovative design concepts and technologies for preclinical PET imaging [2].

The image quality and quantitative accuracy of PET images are degraded by many physical factors such as the attenuation of photons, the detection of scattered photons and the finite spatial resolution of the imaging system. Scattered events decreases image contrast by misplacing events while assigning to a line of response, thus causing bias resulting in the overestimation of the actual activity concentration. The scatter fraction (SF), defined as the ratio of scattered coincidences to total coincidences, is a useful parameter to assess the magnitude of scatter and to estimate its impact on reconstructed PET images. Likewise, the noise equivalent count rate (NECR) is an important parameter used for performance characterization of PET systems [3]. The SF largely depends on object size and density, energy window settings and scanner geometry. The magnitude of SF has been widely studied and is well documented for clinical PET imaging. It represents $10 \%$ to $20 \%$ in twodimensional (2D) mode, whereas it reaches $30-35 \%$ in brain imaging and 50-60\% in whole-body imaging in threedimensional (3D) acquisition mode [4]. Though the characterization of the scatter component in small animal PET imaging has received little attention owing to the small SF, SF estimates have been reported for various small animal PET scanners [5]. Depending on PET system characteristics and acquisition parameters, the typical range is $5 \%$ to $21 \%$ for mice and $15 \%$ to $30 \%$ for rats [6-13]. The SF and NECR are usually measured using various discrete phantoms of different uniform size [14-16]. For instance, the National Electrical Manufacturers Association (NEMA) standards [17] suggest three different phantoms for SF and count rate measurements for small animal PET scanners. These correspond to mouse-, rat- and monkey-sized phantoms of 25, 50 and $100 \mathrm{~mm}$ diameter, respectively. However, within a specific rodent species, especially rats or small rabbits, there is substantial variation in body size when rodents are litters or correspond to diabetic models put on high calorie diets. Also, the rodents' body shape is not uniform throughout in the axial direction. The body cross-section of rodents' specially rat and large species increases from head towards pelvis region. Moreover, it has been suggested that a phantom representing a varying range of cross-sections and dimensions would be more suited for the assessment of these parameters for clinical PET systems [18]. Furthermore, it is nowadays common practice to increase the throughput of rodent PET studies by simultaneous scanning of multiple rodents placed at different radial offsets in the scanner's fields of view (FOV) [19].

The purpose of this study is, first, to design and develop a cone-shaped phantom for the measurement of object sizedependent SF and NECR and, second, to assess these parameters as function of radial offset, object size and lower energy threshold (LET) for two small animal PET scanners, namely the X-PET ${ }^{\mathrm{TM}}$ and LabPET- $8^{\mathrm{TM}}$ using the developed cone-shaped phantom.

\section{Materials and Methods}

\section{Modeling and Validation of Small Animal PET Scanners}

Both the X-PETTM and LabPET- $8^{\mathrm{TM}}$ scanners were modeled as realistically as possible in terms of geometry, physics of photon transport and signal processing using the GATE Monte Carlo simulation toolkit [20]. This package is based on the wellestablished Geant4 libraries [21] featuring a modular, versatile, scripted simulation toolkit. As such, it allows accurate modeling of detailed scanner geometries and radiation interaction with matter, tracking events (recording position, time and energy of an event) and creating various types of output files including projection data and other relevant parameters. Simulation models of both scanners were validated through comparison to experimental measurements, namely sensitivity, NECR and SF for mouse and rat-sized phantoms.

The X-PET ${ }^{\mathrm{TM}}$ and LabPET-8 ${ }^{\mathrm{TM}}$ small animal PET scanners commercialized by GE Healthcare (Waukesha, WI) were used in this work. The X-PETTM is a $\mathrm{Bi}_{4} \mathrm{Ge}_{3} \mathrm{O}_{12}$ (BGO)-based small animal PET scanner, having an axial FOV of $11.6 \mathrm{~cm}[22,23]$. The system incorporates various technological innovations to improve system performance [7]. For instance, the photomultiplier-quadrant-sharing method is used to maximize the number of crystals per photomultiplier whereas a high-yield pileup event recovery electronic processing technology is exploited to improve the count-rate performance [24]. The detector blocks are circularized by slightly grinding each block on the photomultiplier side into a pentagon shape. On the other hand, the LabPET- $8^{\text {TM }}$ is an avalanche photodiode (APD)-based digital PET scanner having a $7.5 \mathrm{~cm}$ axial FOV, designed with quasi-individual crystal readout along with parallel digital architecture to achieve high-performance [11]. Scintillation crystals composed of $\mathrm{Lu}_{0.4} \mathrm{Gd}_{1.6} \mathrm{SiO}_{5}$ (LGSO) and $\mathrm{Lu}_{1.9} \mathrm{Y}_{0.1} \mathrm{SiO}_{5}$ (LYSO) are optically coupled one after the other, 
forming phoswich pairs of detectors that are read out by a single APD. Four phoswich detectors are enclosed in a hermetic container made of kovar (an iron-nickel-cobalt alloy with a density of $8.359 \mathrm{~g} / \mathrm{cm}^{3}$ ) having external dimensions of $10.3 \times 4.7 \times 18 \mathrm{~mm}$. The end of axial FOV shielding is made of tungsten $\left(19.3 \mathrm{~g} / \mathrm{cm}^{3}\right.$ density, $15.75 \mathrm{~mm}$ thickness and $131 \mathrm{~mm}$ internal diameter) to minimize the detection of out-of-FOV activity [11]. The most relevant design features of both scanners are summarized in Table 1.

For the X-PETTM, pentagonal detector blocks were modeled using trapezoidal volumes. An energy resolution of $25 \%$ for $511 \mathrm{keV}$ photons was applied as blurring kernel for energies deposited within the crystals [25]. The LET was set to $250 \mathrm{keV}$ whereas the higher energy threshold was set to $750 \mathrm{keV}$ in all simulations. These energy thresholds were chosen to mimic default energy threshold settings on the actual X-PET ${ }^{\mathrm{TM}}$ scanner.

For the LabPET-8 ${ }^{\mathrm{TM}}$, the GATE materials database was modified to add LYSO, LGSO and kovar. For both LYSO and LGSO, an energy resolution of $25 \%$ and an average timing resolution of 9 ns were set [26]. The coincidence window was set to $20 \mathrm{~ns}$. The LET was set to $250 \mathrm{keV}$ whereas the higher energy threshold was set to $650 \mathrm{keV}$. These energy thresholds and coincidence window settings are similar to the settings on the actual LabPET-8 ${ }^{\mathrm{TM}}$ scanner.

Back-to-back $511 \mathrm{keV}$ annihilation photons were generated to decrease computational time. The animal positioning bed was not modeled for both scanners. The GATE output information was recorded in ASCII format and then rebinned into sinograms using single-slice rebinning (SSRB) [27].

The validation parameters are calculated in according to NEMA-04 procedures. The SF and NECR were calculated for mouse and rat phantoms using radioactive line sources radially offset by 10 and $17.5 \mathrm{~mm}$, respectively. In brief, for each total events sinogram, all pixels located farther than $8 \mathrm{~mm}$ from the edge of the phantom were set to zero. The profile of each projection angle was shifted so that the maximum valued pixels were aligned with the central pixel of the sinograms. A sum projection was obtained by adding up all angular projections in each slice. A linear interpolation between the left and right edges of the $14 \mathrm{~mm}$ central band was used to differentiate the trues from other counts.

The total event rate $R_{\mathrm{tot}, i}$, for each acquisition of each slice $i$ is computed as:

$$
R_{\mathrm{tot}, i}=\frac{C_{\mathrm{tot}, i}}{T_{\mathrm{acq}}}
$$

Table 1. Summary of technical specifications of the X-PET ${ }^{\mathrm{TM}}$ and LabPET$8^{\mathrm{TM}}$ preclinical PET scanners

\begin{tabular}{lll}
\hline Type & X-PET $^{\mathrm{TM}}$ & LabPET-8 $^{\mathrm{TM}}$ \\
\hline Scintillator & BGO & $\begin{array}{c}\text { Phoswich pair of } \\
\text { LYSO/LGSO }\end{array}$ \\
Crystal dimension & $2.32 \times 2.32 \times$ & $\begin{array}{c}2.0 \times 2.0 \times \\
\end{array}$ \\
No. of detector rings & $9.4 \mathrm{~mm}^{3}$ & $14 \mathrm{~mm}^{3}$ \\
Crystals per ring & 48 & 32 \\
Total no. of crystals & 240 & 192 \\
Detector ring inner diameter (mm) & 165 & 6,144 \\
Transaxial field-of-view (mm) & 100 & 162 \\
Axial field-of-view (mm) & 116 & 100 \\
Image pixel size (mm) & 0.4 & 75 \\
Slice thickness (mm) & 0.4 & 0.5 \\
\hline
\end{tabular}

where $T_{\text {acq }}$ is the acquisition time. The system total event rate, $R_{\text {tot }}$, is computed as the sum of $R_{\mathrm{tot}, i}$ over all slices $i$.

The SF for each acquisition was computed using the following formula:

$$
\mathrm{SF}=\frac{R_{\text {scatter }}}{R_{\text {true }}+R_{\text {scatter }}}
$$

The NECR, $R_{\text {nec }}$, for each acquisition was computed as:

$$
R_{\text {nec }}=\frac{R_{\text {true }}^{2}}{R_{\text {total }}}
$$

The peak absolute sensitivity was measured using the ${ }^{22} \mathrm{Na}$ point source as described in NEMA-04 procedures and is given by:

$$
S_{\mathrm{a}}=\frac{S_{\mathrm{i}}}{0.906} \times 100
$$

where 0.906 is the branching ratio of ${ }^{22} \mathrm{Na}$ and $S_{\mathrm{i}}$ is the sensitivity (in counts/s/Bq). The data were processed using programs developed in-house implemented in MATLAB 7.4 (Mathworks, Natick, MA).

The X-PET ${ }^{\mathrm{TM}}$ simulation model was validated against experimental results [7] whereas the LabPET- ${ }^{\mathrm{TM}}$ simulation model was validated against experimental results performed by our group and those reported by Bergeron et al. [28] in terms of sensitivity, NECR and SF for mouse and rat-sized phantoms. The relative difference is calculated as the percentage difference relative to the mean.

\section{Design and Fabrication of the Cone-Shaped Phantom}

Typical shapes of mouse and rat bodies represented by non-uniform rational B-spline (NURBS)-based mouse [29] and rat [30] models are shown in Fig. 1. It can be seen that the upper part of rodent bodies bears a resemblance to a tapered than a cylindrical shape. The parameters considered for phantom design are material composition, cross-sectional dimensions and taper angle $(\theta)$. The fabrication material chosen is high density polyethylene (density of $\left.0.96 \pm 0.1 \mathrm{~g} / \mathrm{cm}^{3}\right)$ as prescribed in the NEMA-NU04 standards. The maximum diameter of the phantom was derived from the transaxial FOV of various small animal scanners $[6,7,9,11,31]$. Based on this assessment, the maximum diameter of the phantom was set to $70 \mathrm{~mm}$ by considering $70 \%$ of the average maximum diameter of the transaxial FOV. The minimum diameter of the phantom was set to $20 \mathrm{~mm}$ because of limitations of the milling machine used. The length of the phantom depends on the taper angle. The length $L$ and the taper angle $\theta$ are related by the following equation:

$L=($ Maximum diameter - minimum diameter $) /(2 \times \tan \theta)$

The corresponding phantom length is 28.6, 20.4, 15.8 and $12.9 \mathrm{~cm}$ for taper angles of $5^{\circ}, 7^{\circ}, 9^{\circ}$ and $11^{\circ}$. As $\theta$ increases, the phantom length decreases, thus increasing the range of the crosssectional volume covering one axial FOV, making it less 


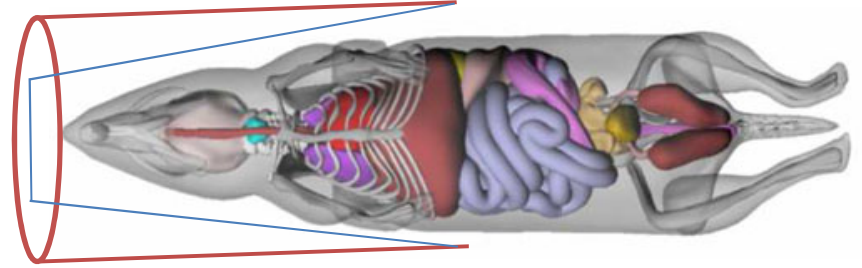

(a)

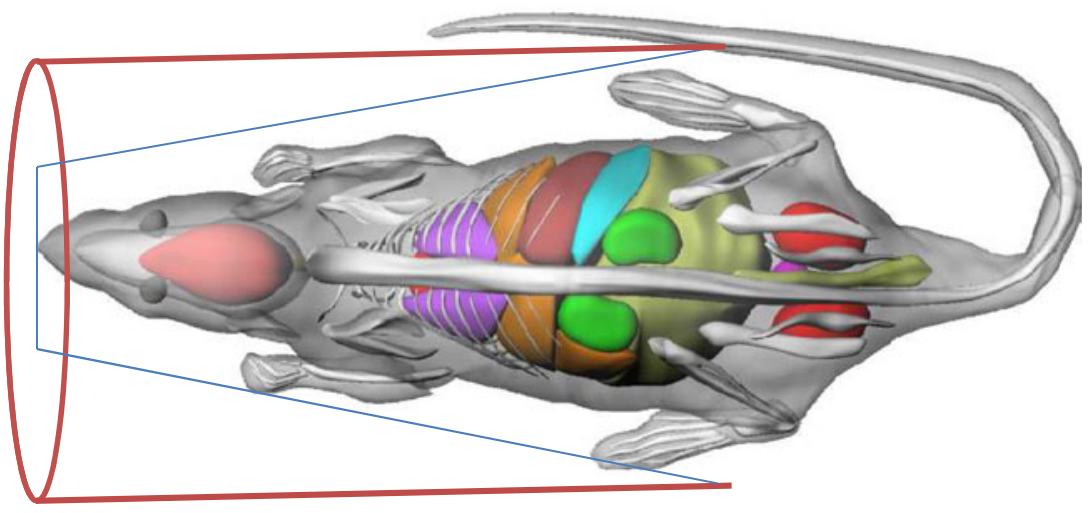

(b)

Fig. 1. Illustration of rodents' shape in the axial direction using NURBS-based preclinical anatomical models corresponding to $\mathbf{a}$ mouse and $\mathbf{b}$ rat.

representative of an equivalent volume cylindrical (EVC) phantom. However, for small $\theta$, the phantom becomes long enough for the corresponding small animal PET axial FOV. The EVC phantom is defined as a phantom having a uniform cylindrical shape and diameter equal to the diameter of the cone-shaped phantom in the middle of the axial FOV of the scanner (Fig. 2). When $\theta=0^{\circ}$, the cone-shaped phantom corresponds to the EVC phantom having diameter of minimum or maximum side of the cone-shaped phantom. Therefore, the optimization of the taper angle is crucial for the design of the cone-shaped phantom. Different cone-shaped phantoms with minimum diameter of $2 \mathrm{~cm}$, maximum diameter of $7 \mathrm{~cm}$ and taper angles of $5^{\circ}, 7^{\circ}, 9^{\circ}$ and $11^{\circ}$ were simulated using the Monte Carlo model of the X-PET ${ }^{\mathrm{TM}}$ scanner described in "Modeling and Validation of Small Animal PET Scanners". Line sources of different length were placed in the center of the phantom. Simulations were performed for different taper angles of $5^{\circ}, 7^{\circ}, 9^{\circ}$ and $11^{\circ}$ and its corresponding EVC phantom for central region of phantom. The corresponding diameter of the EVC phantom for taper angles of $5^{\circ}, 7^{\circ}, 9^{\circ}$ and $11^{\circ}$ were $5.5,5,4.5$ and $4 \mathrm{~cm}$, respectively. The taper angle was optimized by analyzing the simulated data of the X-PET ${ }^{\mathrm{TM}}$ scanner in terms of SF, NECR and true count rate using the NEMA-NU04 standards as described in "Modeling and Validation of Small Animal PET Scanners".

The phantom was fabricated in-house considering the optimized design considerations discussed above including material, crosssectional dimensions and taper angle. To study the effects of radial offsets, four holes (4 $\mathrm{mm}$ diameter) were drilled parallel to the long axis at the center and at radial offset positions of 10, 15 and $20 \mathrm{~mm}$. Solid line fillings of the same material used for the phantom were also fabricated. These fillings were used to fill up the remaining holes when one particular hole is filled with a radioactive line source during acquisition.
The different FOVs of the cone-shaped phantom corresponding to minimum, middle and maximum diameter region in the scanner's axial FOV will be referred to as $\mathrm{FOV}_{\text {mouse, }} \mathrm{FOV}_{\text {rat }}$ and $\mathrm{FOV}_{\text {rabbit }}$, respectively. The phantom dimensions of each FOV for the X-PET ${ }^{\mathrm{TM}}$ and LabPET- $8^{\mathrm{TM}}$ scanners are summarized in Table 2. The cone-shaped phantom can be dismantled into three equal parts, thus allowing various combinations to match the axial FOV of different small animal PET scanners. Depending on the scanner's axial FOV, the cone-shaped phantom can be exploited to simulate various sizes of mice, rats and small rabbits used in small animal PET imaging. For instance, in the case of the LabPET- $8^{\mathrm{TM}}$, $\mathrm{FOV}_{\text {mouse }}$ (diameter from 2 to $4.5 \mathrm{~cm}$ ), $\mathrm{FOV}_{\text {rat }}$ (diameter from 3 to $6 \mathrm{~cm}$ ) and $\mathrm{FOV}_{\text {rabbit }}$ (diameter from 4.5 to $7 \mathrm{~cm}$ ), can be used to simulate the various sizes of these species to assess variations in $\mathrm{SF}$ and NECR.

\section{Studies using the Cone-Shaped Phantom}

The optimized cone-shaped phantom was further used to assess the magnitude of scatter and NECR as function of varying phantom size, LET and line source position for both small animal PET scanners using both Monte Carlo simulation and experimental studies.

Simulation Studies PET data were simulated and analyzed for a line source at the center and at radial offsets of 10,15 and $20 \mathrm{~mm}$ for LETs of 250, 350 and $425 \mathrm{keV}$, whereas the higher energy threshold was kept constant $\left(650 \mathrm{keV}\right.$ for LabPET-8 ${ }^{\mathrm{TM}}$ and $750 \mathrm{keV}$ for the X-PETTM). Back-to-back $511 \mathrm{keV}$ annihilation photons were simulated for one line source at a time $\left(10^{9}\right.$ events). 

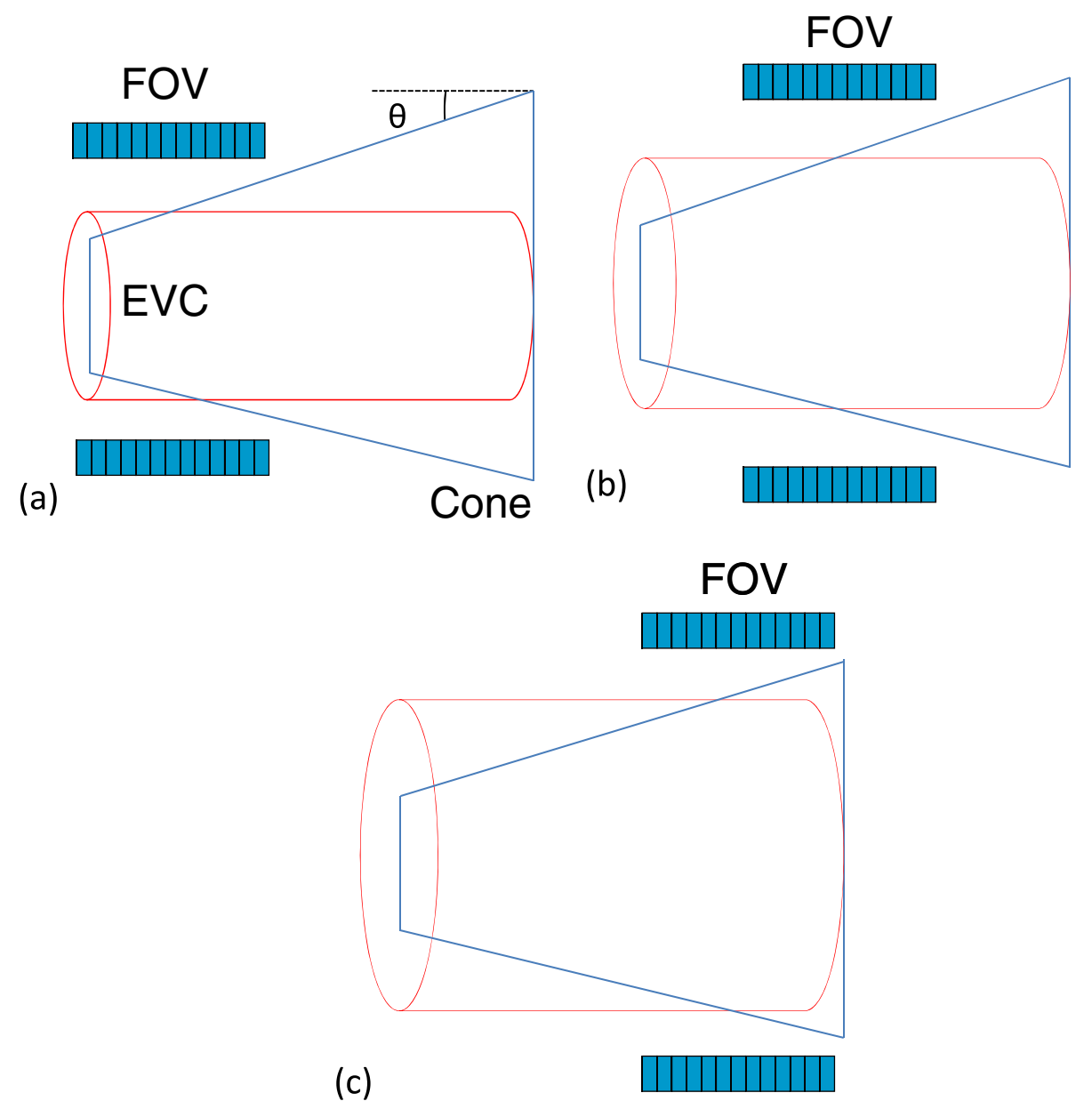

Fig. 2. Illustration of the cone-shape phantom and its equivalent volume cylindrical (EVC) phantom for the various axial fieldsof-view: a minimum ( $\left.\mathrm{FOV}_{\text {mouse }}\right)$, b middle $\left(\mathrm{FOV}_{\text {rat }}\right)$ and $\mathbf{c}$ maximum ( $\mathrm{FOV}_{\text {rabbit }}$ ) diameter region in the scanner's axial FOV.

All simulations were performed at a low radioactivity regime as defined in the NEMA-04 standards for SF calculation. Data were collected at three different axial FOVs from one end of the phantom to the other in three successive axial steps, namely $\mathrm{FOV}_{\text {mouse }}$, $\mathrm{FOV}_{\text {rat }}$ and $\mathrm{FOV}_{\text {rabbit }}$ An EVC of the cone-shaped phantom at three different axial FOVs was also simulated.

Experimental Studies Simulation studies using the cone-shaped phantom were experimentally validated for the LabPET-8 ${ }^{\mathrm{TM}}$ scanner. The line source was filled with $2 \mathrm{MBq}$ of ${ }^{18} \mathrm{~F}$ and inserted in the central hole of the phantom while the other holes were closed using the fillings. The phantom was placed on the scanner bed using Styrofoam support at the smaller end of the phantom. Adhesive tape was also used to secure the phantom on the scanner bed. The data were acquired for three FOVs, namely FOV corrected for radioactive decay. This was repeated for other holes and always a new line source filled with $2 \mathrm{MBq}$ of ${ }^{18} \mathrm{~F}$ was used. Each acquisition lasted $5 \mathrm{~min}$. PET studies were acquired using a default energy window of $250-650 \mathrm{keV}$ and $10 / 15 / 20 \mathrm{~ns}$ time window for LYSO-LYSO/LYSO-LGSO/ LGSO-LGSO coincidences in list-mode format which were binned into 3D sinograms. These 3D sinograms were further

Table 2. Dimensions of different FOVs of the cone-shaped and EVC phantoms for the X-PET ${ }^{\mathrm{TM}}$ and LabPET-8 ${ }^{\mathrm{TM}}$ scanners

\begin{tabular}{|c|c|c|c|c|c|c|}
\hline \multirow[t]{2}{*}{ Scanner } & \multirow[t]{2}{*}{ FOV } & \multicolumn{3}{|l|}{ Cone-shaped phantom } & \multicolumn{2}{|l|}{ EVC phantom } \\
\hline & & Min. diameter $(\mathrm{cm})$ & Max. diameter $(\mathrm{cm})$ & Length (cm) & Diameter (cm) & Length $(\mathrm{cm})$ \\
\hline \multirow[t]{3}{*}{ X-РET ${ }^{\text {TM }}$} & $\mathrm{FOV}_{\text {mouse }}$ & 2 & 6 & 11.6 & 4 & 11.6 \\
\hline & $\mathrm{FOV}_{\text {rat }}$ & 2.5 & 6.5 & 11.6 & 4.5 & 11.6 \\
\hline & $\mathrm{FOV}_{\text {rabbit }}$ & 3 & 7 & 11.6 & 5.0 & 11.6 \\
\hline \multirow[t]{3}{*}{ LabPET- $8^{\text {TM }}$} & $\mathrm{FOV}_{\text {mouse }}$ & 2 & 4.5 & 7.5 & 3.2 & 7.5 \\
\hline & $\mathrm{FOV}_{\text {rat }}$ & 3 & 6 & 7.5 & 4.5 & 7.5 \\
\hline & $\mathrm{FOV}_{\text {rabbit }}$ & 4.5 & 7 & 7.5 & 5.8 & 7.5 \\
\hline
\end{tabular}




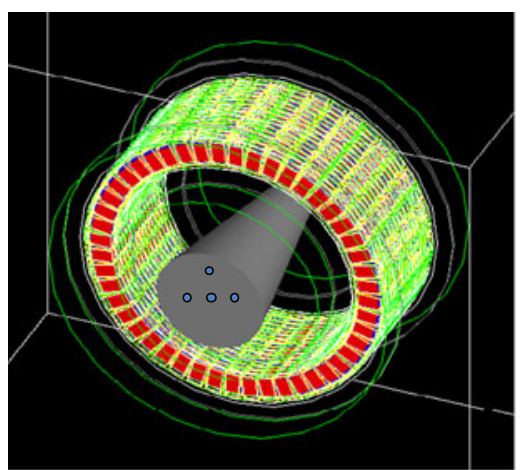

(a)

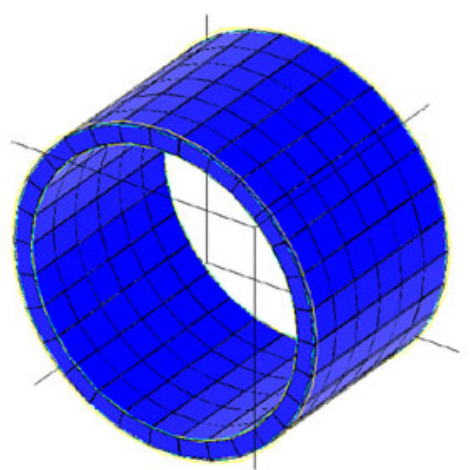

(b)

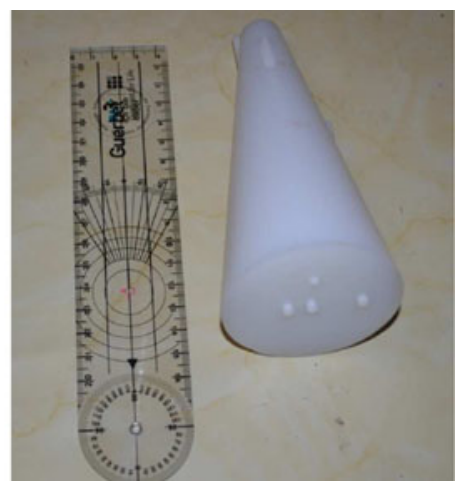

(c)

Fig. 3. Simulation models using GATE of $\mathbf{a}$ the LabPET-8 ${ }^{\mathrm{TM}}$ scanner and $\mathbf{b}$ the X-PET ${ }^{\mathrm{TM}}$ scanner. $\mathbf{c}$ Photograph of the fabricated cone-shaped physical phantom.

rebinned to $2 \mathrm{D}$ sinograms using $\mathrm{SSRB}$. These $2 \mathrm{D}$ sinograms were used for further analysis adhering to NEMA-NU04 standards. The relative difference (in percent) between simulated and experimental results was also calculated.

\section{Results}

\section{Cone-Shaped Phantom}

Figure 3a-b illustrates the modelled LabPET- $8^{\mathrm{TM}}$ and X-PET ${ }^{\mathrm{TM}}$ using GATE toolkit. The developed physical cone-shaped phantom is shown in Fig. 3c. The optimal minimum and maximum diameters of the cone-shaped phantom are 2 and $7 \mathrm{~cm}$, respectively. The total and true events rate, NECR, SF and total phantom length for different taper angles of the cone-shaped phantom are summarized in Table 3. Out of four simulated taper angles, an angle of $11^{\circ}$ produced the highest total, true and NECR events rate whereas it resulted in the lowest SF. Figure 4 presents the SF profile for slice numbers covering the axial FOV of the X-PET ${ }^{\mathrm{TM}}$ scanner. It can be observed that the SF profiles for $9^{\circ}$ and $11^{\circ}$ are closer to the SF profile of the EVC phantom. Since a maximum taper angle of $10^{\circ}$ was not possible for the milling machine used to fabricate the phantom due to its limitations, a taper angle of $9^{\circ}$ was chosen.

\section{Experimental and Simulation Studies}

The validation results of the simulated X-PETTM and LabPET- $8^{\mathrm{TM}}$ models are summarized in Table 4 . It can be seen that there is good agreement between simulated and measured values for all considered parameters with a relative difference varying between $4.8 \%$ and $10.8 \%$. This gives confidence to use the simulated models for prediction of performance parameters under various conditions. For Monte Carlo simulation model validation, the line source position was set according to NEMA-NU04 standards, that is, $10 \mathrm{~mm}$ for the mouse phantom and $17.5 \mathrm{~mm}$ for the rat phantom, radially offset from the center.

Experimental studies performed using the cone-shaped phantom were acquired on the LabPET- $8^{\mathrm{TM}}$ scanner. Figure 5 shows the percent relative difference between simulated and experimental results for the LabPET- $8^{\mathrm{TM}}$ scanner in terms of NECR and SF as function of line source radial position for the three considered FOVs $\left(\mathrm{FOV}_{\text {mouse }}, \mathrm{FOV}_{\text {rat }}\right.$ and $\left.\mathrm{FOV}_{\text {rabbit }}\right)$. The resulting relative differences using an energy window of $250-650 \mathrm{keV}$ vary mostly between $0.7 \%$ and $10 \%$ and overall remain below $16 \%$.

The $\mathrm{SF}$ for $\mathrm{FOV}_{\text {mouse }}$ corresponding to the cone-shaped and EVC phantoms for both the X-PET ${ }^{\mathrm{TM}}$ and LabPET-8 ${ }^{\mathrm{TM}}$ scanners using a LET of 250,350 and $425 \mathrm{keV}$ is shown in Fig. 6 . The SF estimates are shown for a line source located at the center and at 10 and $15 \mathrm{~mm}$ radial offset. Similar results are shown in Fig. 7 for $\mathrm{FOV}_{\text {rabbit. }}$ In the former instances, a line source with radial offset of $20 \mathrm{~mm}$ was not considered since the minimum diameter for $\mathrm{FOV}_{\text {mouse }}$ is $20 \mathrm{~mm}$. Using the cone-shaped phantom, the SF for $\mathrm{FOV}_{\text {rat }}$ varies as a function of the radial offset of the line source. The range of this variation is from $26.3 \%$ to $18.2 \%, 18.6$ to $13.1 \%$ and 10.1 to $7.6 \%$ for the X-PET ${ }^{\mathrm{TM}}$, whereas it was from $34.4 \%$ to $26.9 \%, 19.1$ to $17.0 \%$ and 9.1 to $7.3 \%$ for the

Table 3. Summary of total and true events rate, NECR and SF for different taper angles of the cone-shaped phantom for the X-PETTM scanner

\begin{tabular}{llllll}
\hline Taper angle $(\theta)$ & Total events rate & True events rate & NECR & SF & Phantom length $(\mathrm{cm})$ \\
\hline $11^{\circ}$ & 31,594 & 24,024 & 18,329 & 23.96 & 12.9 \\
$9^{\circ}$ & 31,194 & 22,957 & 16,933 & 26.41 & 15.8 \\
$7^{\circ}$ & 30,734 & 21,932 & 15,677 & 28.64 & 20.4 \\
$5^{\circ}$ & 30,388 & 21,152 & 14,741 & 30.39 & 28.6 \\
\hline
\end{tabular}




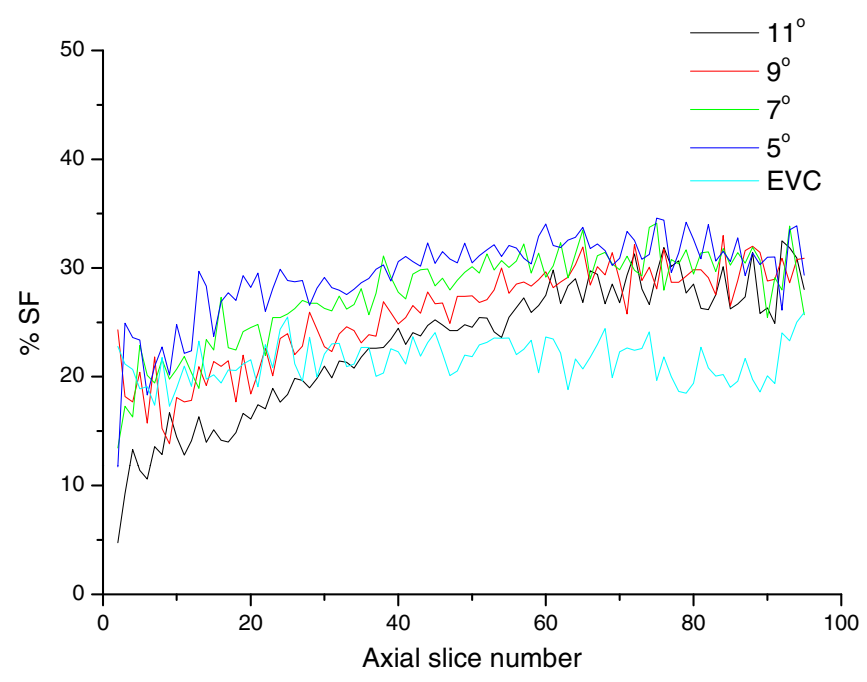

Fig. 4. Plots of SF (in percent) versus axial slices for different taper angles of the cone-shaped phantom and equivalent cylindrical volume (EVC) phantom.

LabPET-8 ${ }^{\mathrm{TM}}$, for LETs of 250, 350 and $425 \mathrm{keV}$, respectively. For the EVC phantom, the SF varied from $21.8 \%$ to $15.5 \%, 17.0$ to $11.3 \%$ and 10.7 to $7.6 \%$ for the X-PET ${ }^{\mathrm{TM}}$, whereas it varied from $29.8 \%$ to $23.8 \%, 18.6$ to $14.4 \%$ and 9.1 to $7.0 \%$ for the LabPET-8 ${ }^{\mathrm{TM}}$, for LETs of 250,350 and $425 \mathrm{keV}$, respectively.

Figure 8 shows the NECR for the LabPET- $8^{\text {TM }}$ scanner for both the cone-shaped and EVC phantoms for three axial FOVs versus the line source radial offset when using LETs of 250,350 and $425 \mathrm{keV}$. The same parameter is plotted for the X-PET ${ }^{\mathrm{TM}}$ scanner in Fig. 9. Table 5 highlights the high correlation $\left(R^{2}\right)$ between the SF and NECR estimates for the cone-shaped phantom and the EVC phantom for the three axial FOVs for both scanners.

As expected, the SF for both scanners decreases as the radial offset increases, LET increases and object size decreases. However, in all cases the SF of the LabPET$8^{\mathrm{TM}}$ is higher than the X-PET ${ }^{\mathrm{TM}}$ scanner. The NECR for both scanners increases as the radial offset increases and object size decreases. The NECR reached a maximum for the LabPET-8 ${ }^{\text {TM }}$ at a LET of $350 \mathrm{keV}$ whereas it reached the maximum value at a LET of $250 \mathrm{keV}$ for the $\mathrm{X}$ PETTM. However, in all cases the NECR for the XPET ${ }^{\mathrm{TM}}$ is higher compared with the LabPET- $8^{\mathrm{TM}}$ scanner.

\section{Discussion}

The SF and NECR are important parameters for optimization of acquisition protocol settings such as timing and energy windows and comparing the performance of different small animal PET scanners. In addition, the SF is useful for the assessment of the relevance and level of complexity of scatter correction required for small animal PET studies. These parameters are usually measured using various discrete uniform phantoms of different size [14-16]. Reproducibility of measurements and shape/size of the phantoms are some limitations of this approach. The analysis of body shape of various voxel-based, NURBS-based models and actual laboratory animals revealed that the shape of the upper body of rodents resembles more to tapered shape than cylindrical shape (Fig. 1). It should also be emphasized that there is considerable variation in body size within particular rodents' species, especially rats and small rabbits. This motivated the design and fabrication of a single cone-shaped phantom suitable for assessing object size-dependent SF and NECR of small animal PET systems. It should be noted that the purpose is not to replace or suggest an alternative to well established NEMA standards [17]. The phantom design was optimized using Monte Carlo simulation studies of the XPET $^{\mathrm{TM}}$ scanner using the GATE toolkit. The validation of simulation models of both scanners against experimental measurements proved that the models are capable of predicting the response of actual systems with acceptable accuracy. Overall, there was good agreement between simulated and experimental results for both scanners, with a relative error varying between $4.8 \%$ and $10.8 \%$. The methodology followed for the design and optimization of the cone-shaped phantom is an extension of the work described by Wilson et al. [18] in the context of clinical PET to small animal imaging. The phantom described in the reference above is a fillable tapered design suitable for clinical imaging, whereas we opted for a solid tapered phantom dedicated for small animal PET imaging having multiple holes to insert line sources at different radial offset positions.

The phantom design parameters that had to be optimized are minimum and maximum diameters, length of the cone and taper angle. Although the determined optimal taper angle is $11^{\circ}$, a taper angle of $9^{\circ}$ was used for fabricating the phantom owing to the limitations of the milling machine. Using the cone-shaped phantom, the difference between

Table 4. Comparison between simulated and measured performance parameters of the X-PET ${ }^{\mathrm{TM}}$ and LabPET-8 ${ }^{\mathrm{TM}}$ small animal PET scanners

\begin{tabular}{|c|c|c|c|c|c|c|}
\hline \multirow[t]{2}{*}{ Parameter } & \multicolumn{3}{|l|}{ X-PET ${ }^{\mathrm{TM}}$} & \multicolumn{3}{|c|}{ LabPET-8 $8^{\mathrm{TM}}$} \\
\hline & Simulated & Measured $^{\mathrm{a}}$ & Relative difference $(\%)$ & Simulated & Measured $^{\mathrm{b}}$ & Relative difference $(\%)$ \\
\hline SF (\%) mouse phantom & 7.2 & 7.9 & 9.2 & 18.10 & 19 & 4.8 \\
\hline SF (\%) rat phantom & 19.1 & 21 & 9.4 & 28.18 & 31 & 9.5 \\
\hline Peak NECR (kcps) mouse phantom & 114 & 106 & 7.2 & 204 & 183 & 10.8 \\
\hline Absolute Sensitivity (\%) & 6.3 & 5.9 & 6.5 & 1.46 & 1.33 & 9.3 \\
\hline
\end{tabular}

${ }^{\text {a } D a t a}$ taken from Ref. [7]

${ }^{\mathrm{b}}$ Data taken from Ref. [28] 

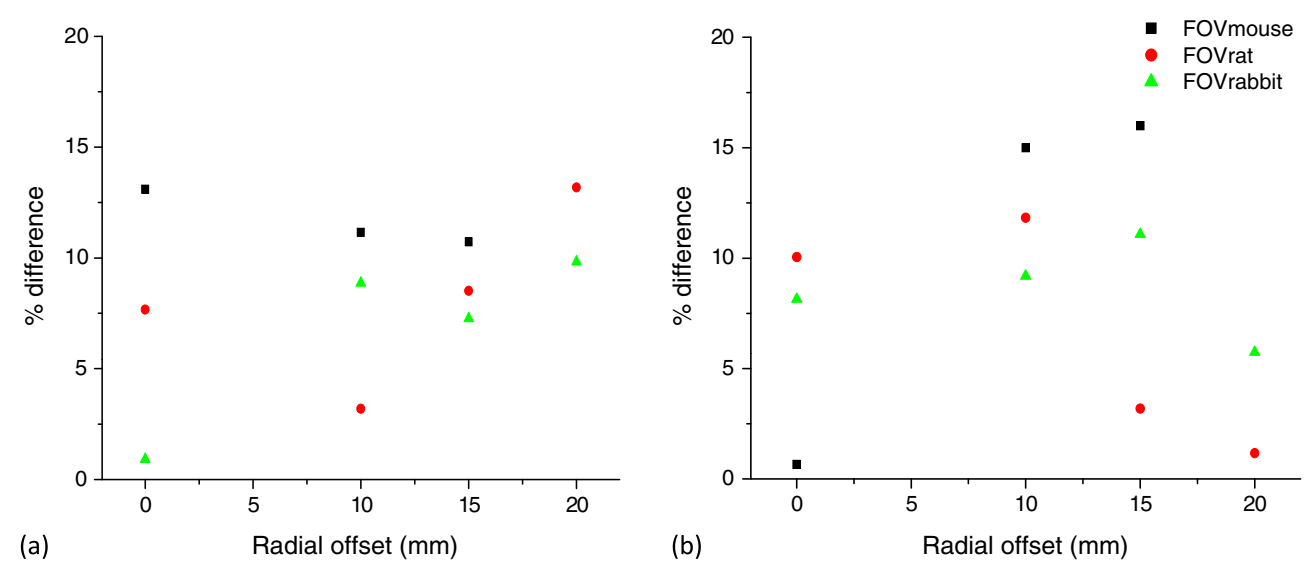

Fig. 5. Relative difference between simulated and experimental results for the LabPET-8 ${ }^{\mathrm{TM}}$ scanner in terms of SF (a) and $\operatorname{NECR}(\mathbf{b})$.

simulated and experimental SF and NECR results for the LabPET- $8^{\text {TM }}$ scanner is below $16 \%$. It should be noted that we have not considered the positron range, animal bed, the intrinsic radioactivity emanating from LGSO and LYSO crystals in the simulation model. Inclusion of positron range in the simulation model can affect the scatter fraction estimates, especially for smaller animal sizes. However, this may not have a significant effect for the low energy positrons of ${ }^{18} \mathrm{~F}$ [16]. On the other hand, Rechka et al. [26] have shown that the Compton scattering probability due to scanner bed is small $(2.8 \%)$. Experimental studies using the cone-shaped phantom could not be performed on the X-PET ${ }^{\mathrm{TM}}$ scanner since it was upgraded to LabPET-8 ${ }^{\mathrm{TM}}$ and as such is no longer available for further investigation.

The SF decreases as the radial offset increases from center to radial offset of $20 \mathrm{~mm}$, LET increases from 250 to $425 \mathrm{keV}$ and, object size decreases from small rabbit body size to mouse body size. The NECR for both scanners increases as the radial offset increases and object size decreases. The maximum NECR is achieved at a LET of $350 \mathrm{keV}$ for the LabPET- $8^{\mathrm{TM}}$ and $250 \mathrm{keV}$ for the X-PETTM. This reflects the effect of radial position, object size and LET on SF and NECR, which in turn affects image quality and

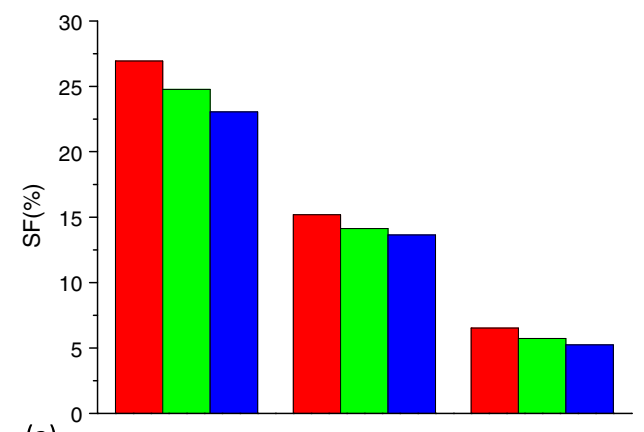

(a)
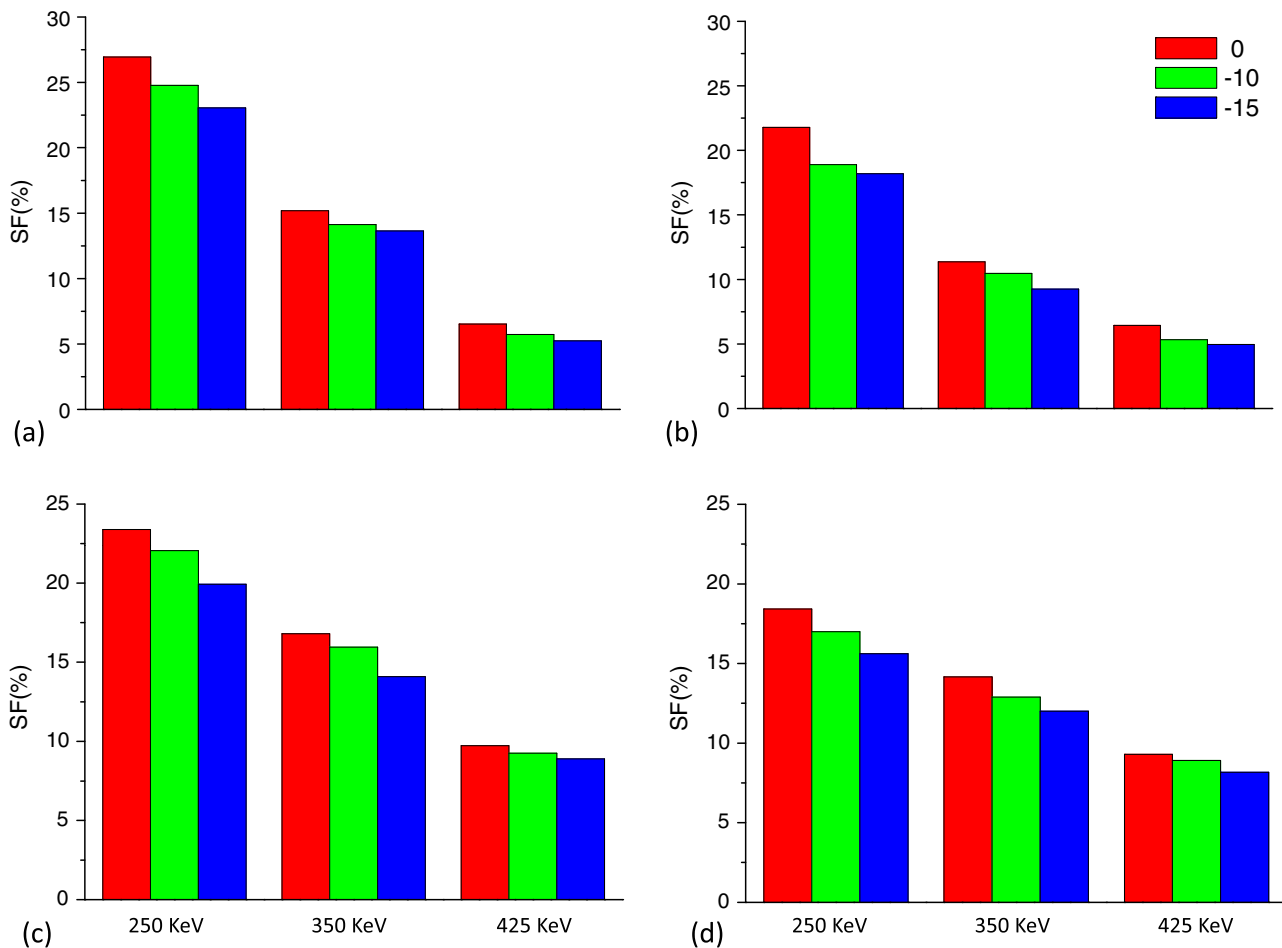

Fig. 6. Variation of SF (in percent) as a function of radial offsets using a LET of 250, 350 and 425 keV for axial FOV mouse. a The LabPET-8 ${ }^{\mathrm{TM}}$ cone-shaped phantom, b LabPET-8 ${ }^{\mathrm{TM}}$ EVC phantom, c X-PET'M cone-shaped phantom, d X-PETTM EVC phantom. 

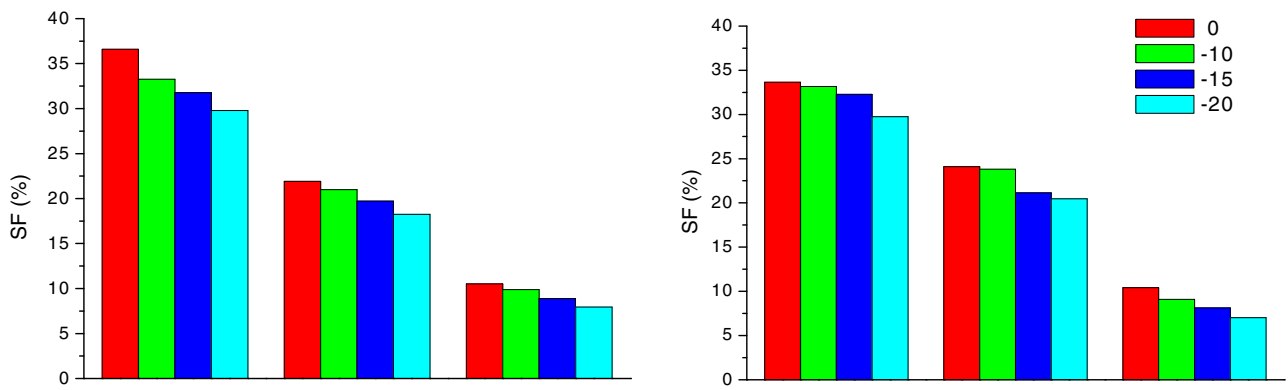

(a)

(b)
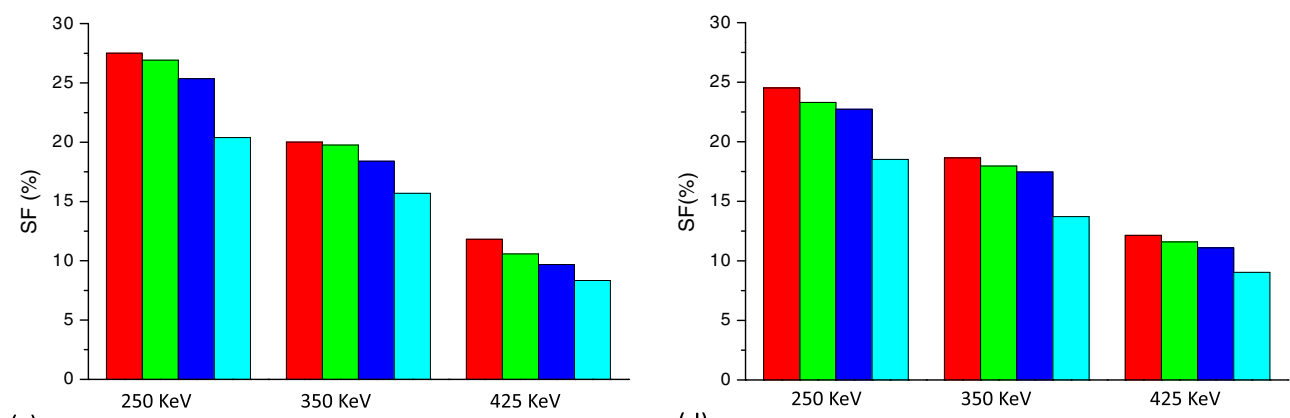

(d)

Fig. 7. Variation of SF (in percent) as a function of radial offsets using a LET of 250, 350 and $425 \mathrm{keV}_{\text {for }}$ axial FOV $\mathrm{Fabbit}$ a The LabPET-8 ${ }^{\mathrm{TM}}$ cone-shaped phantom, b LabPET-8 ${ }^{\mathrm{TM}}$ EVC phantom, c X-PET ${ }^{\mathrm{TM}}$ cone-shaped phantom, d X-PET ${ }^{\mathrm{TM}}$ EVC phantom.

quantitative accuracy of small animal PET imaging. Similar behaviour has been reported elsewhere [14]. The SF for the LabPET- $8^{\text {TM }}$ is higher than for the X-PET ${ }^{\text {TM }}$ when using the same settings. This can be attributed to the detector housing and FOV shielding of the LabPET ${ }^{\mathrm{TM}}$ scanner which increases the signal detection efficiency by $23 \%$ [26], the higher sensitivity to out-of-FOV activity for the LabPET$8^{\mathrm{TM}}$ owing to the shorter axial FOV, inter-crystal scattering
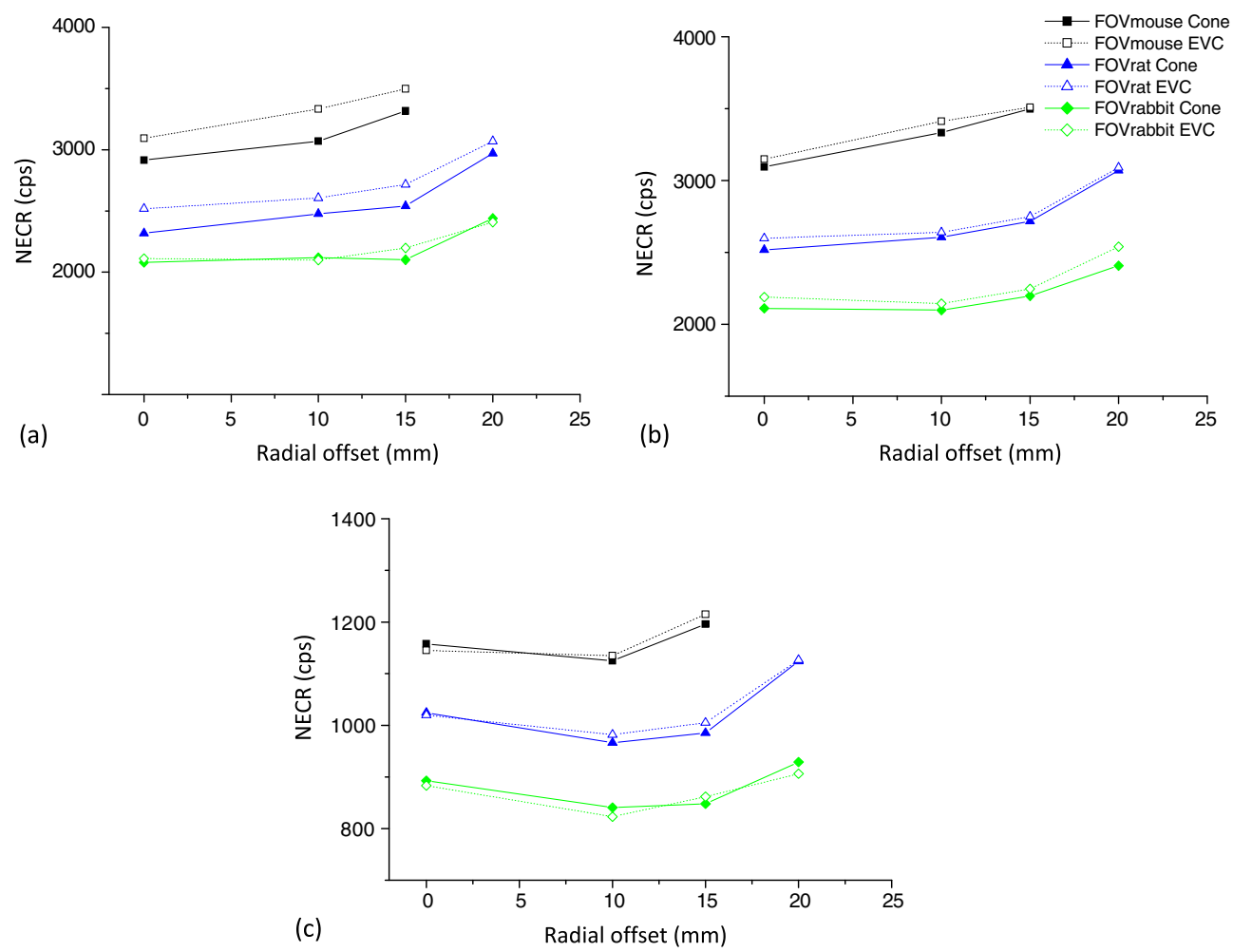

Fig. 8. a Trends of the LabPET-8 ${ }^{\mathrm{TM}}$ NECR as function of radial offsets for the cone-shaped and EVC phantoms using different LETs: a 250 , b 350 and c $425 \mathrm{keV}$. 


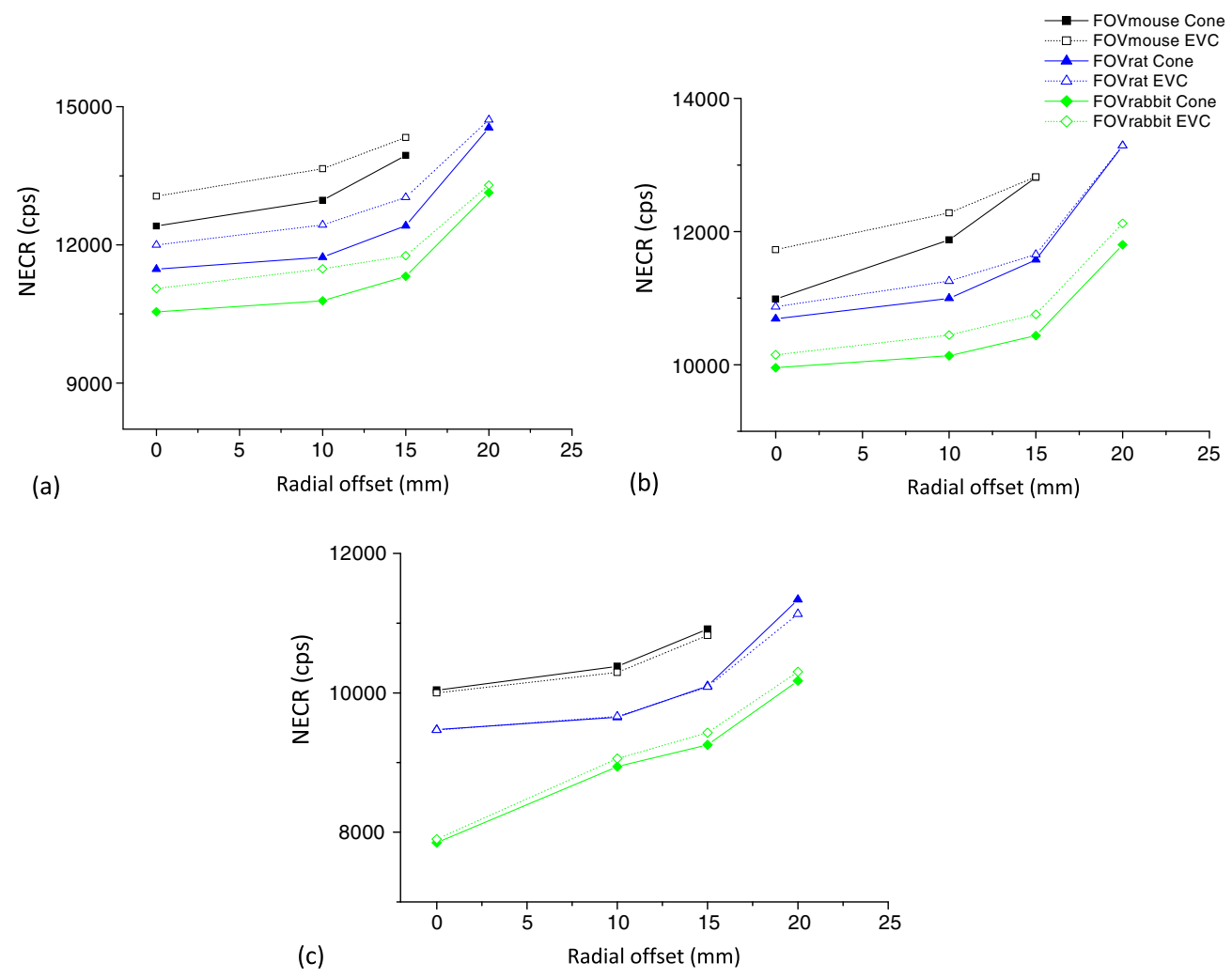

Fig. 9. Trends of the X-PETTM NECR as function of radial offsets for the cone-shaped and EVC phantoms using different LETs: a 250 , b 350 and c $425 \mathrm{keV}$.

which is higher for LYSO/LGSO compared with BGO [32] and the inherent scintillation crystal material characteristics [33]. Moreover, the difference between the SF of the LabPET-8 ${ }^{\mathrm{TM}}$ and X-PET ${ }^{\mathrm{TM}}$ scanners decreases as the LET increases. Overall, the maximum SF observed using the cone-shaped phantom for mouse, rat and small rabbit animals using a LED of $250 \mathrm{keV}$ is $23.3 \%, 34.4 \%$ and $36.5 \%$, respectively. This includes the scatter from objects under study, scanner gantry and surrounding environment. This suggests that scatter correction is important for the accurate quantification in small animal PET imaging [34].

High correlation coefficients for SF and NECR were observed between the cone-shaped phantom and the EVC phantom for different axial FOVs. This seems to indicate that the cone-shaped phantom can be equally used as the uniform EVC phantom to simulate different small animal body sizes. Similar conclusions were drawn by Wilson et al. [18] in the context of clinical PET imaging.

An interesting aspect of the cone-shaped phantom is that it allows the assessment of the effect of three parameters, namely radial offset, energy threshold and object size in a single acquisition. The cone-shaped phantom can be used for evaluation of the effects of radial offsets when imaging several mice simultaneously [19]. Regions corresponding to FOV tom can be used to assess the SF and NECR for various sized animals including mice, rats and small rabbits. Since the cone-shaped phantom can be dismantled into three equal parts, it can be used in various combinations to cope with the axial FOV of different small animal PET scanners.

Table 5. Correlation coefficient $\left(R^{2}\right)$ for NECR and SF between the cone-shaped phantom and EVC phantom for both the X-PETTM and LabPET- $8^{\mathrm{TM}}$ scanners

\begin{tabular}{|c|c|c|c|c|c|c|c|}
\hline \multirow[t]{2}{*}{ Scanner } & \multirow[t]{2}{*}{ LET } & \multicolumn{2}{|c|}{$\mathrm{FOV}_{\text {mouse }}$} & \multicolumn{2}{|l|}{$\mathrm{FOV}_{\text {rat }}$} & \multicolumn{2}{|c|}{$\mathrm{FOV}_{\text {rabbit }}$} \\
\hline & & SF & NECR & SF & NECR & $\mathrm{SF}$ & NECR \\
\hline \multirow[t]{3}{*}{ Х-РET } & 250 & 0.990 & 0.999 & 0.998 & 0.996 & 0.992 & 0.995 \\
\hline & 350 & 0.950 & 0.999 & 0.996 & 0.999 & 0.982 & 0.998 \\
\hline & 425 & 0.965 & 0.999 & 0.895 & 0.996 & 0.951 & 0.960 \\
\hline \multirow[t]{3}{*}{ LabPET-8 $8^{\mathrm{TM}}$} & 250 & 0.963 & 0.973 & 0.999 & 0.995 & 0.885 & 0.946 \\
\hline & 350 & 0.958 & 0.988 & 0.932 & 0.997 & 0.954 & 0.993 \\
\hline & 425 & 0.990 & 0.999 & 0.983 & 0.990 & 0.988 & 0.923 \\
\hline
\end{tabular}




\section{Conclusions}

A cone-shaped phantom was designed and fabricated for assessment of object size-dependent SF and NECR for small animal PET scanners. The characteristics of the LabPET- $8^{\mathrm{TM}}$ and X-PET ${ }^{\mathrm{TM}}$ small animal PET scanners were studied using this phantom in terms of SF and count rate analysis. A single cone-shaped phantom enables the assessment of the effect of three factors, namely radial offset, energy threshold and object size on the SF and NECR for various sizes of mice, rats and small rabbits. This makes the cone-shaped phantom suitable for evaluation of object size-dependent SF and NECR for small animal PET imaging.

Acknowledgements. This work was supported by the Swiss National Science Foundation under grant SNSF 3152A0-102143 and the Indo Swiss Joint Research Programme ISJRP 138866. The authors would like to thank JeanDaniel Leroux and Roger Lecomte from the University of Sherbrooke, Canada, for their help with Monte Carlo modeling of the LabPETTM system.

Conflicts of Interest. All authors declare that they have no conflict of interest.

Open Access. This article is distributed under the terms of the Creative Commons Attribution License which permits any use, distribution, and reproduction in any medium, provided the original author(s) and the source are credited.

\section{References}

1. Chatziioannou AF (2002) Molecular imaging of small animals with dedicated PET tomographs. Eur J Nucl Med Mol Imaging 29:98-114

2. Levin CS, Zaidi H (2007) Current trends in preclinical PET system design. PET Clinics 2:125-160

3. Strother SC, Casey ME, Hoffman EJ (1990) Measuring PET scanner sensitivity: relating countrates to image signal-to-noise ratios using noise equivalents counts. IEEE Trans Nucl Sci 37:783-788

4. Zaidi H, Koral KF (2004) Scatter modelling and compensation in emission tomography. Eur J Nucl Med Mol Imaging 31:761-782

5. Zaidi H, Montandon M-L (2007) Scatter compensation techniques in PET. PET Clinics 2:219-234

6. Bao Q, Newport D, Chen M, Stout DB, Chatziioannou AF (2009) Performance evaluation of the Inveon dedicated PET preclinical tomograph based on the NEMA NU-4 standards. J Nucl Med 50:401408

7. Prasad R, Ratib O, Zaidi H (2010) Performance evaluation of the FLEX Triumph ${ }^{\mathrm{TM}}$ X-PET scanner using the NEMA NU-04 standards. J Nucl Med 51:1608-1615

8. Tai YC, Ruangma A, Rowland D, Siegel S, Newport DF, Chow PL et al (2005) Performance evaluation of the microPET focus: a thirdgeneration microPET scanner dedicated to animal imaging. J Nucl Med 46:455-463

9. Kim JS, Lee JS, Im KC, Kim SJ, Kim S-Y, Lee DS et al (2007) Performance measurement of the microPET Focus 120 scanner. J Nucl Med 48:1527-1535

10. Del Guerra A, Bartoli A, Belcari N, Herbert D, Motta A, Vaiano A et al (2006) Performance evaluation of the fully engineered YAP-(S)PET scanner for small animal imaging. IEEE Trans Nucl Sci 53:1078-1083

11. Bergeron M, Cadorette J, Beaudoin JF, Lepage MD, Robert G, Selivanov V et al (2009) Performance evaluation of the LabPET APD-based digital PET scanner. IEEE Trans Nucl Sci 56:10-16

12. Prasad R, Ratib O, Zaidi H (2011) NEMA NU-04-based performance characteristics of the LabPET- $8^{\text {TM }}$ small animal PET scanner. Phys Med Biol 56:6649-6664
13. Zhang H, Bao Q, Vu N, Silverman R, Taschereau R, Berry-Pusey B et al (2011) Performance evaluation of PETbox: a low cost bench top preclinical PET scanner. Mol Imaging Biol 13:949-961

14. Yang Y, Cherry SR (2006) Observations regarding scatter fraction and NEC measurements for small animal PET. IEEE Trans Nucl Sci 53:127-132

15. Ferrero A, Poon JK, Chaudhari AJ, MacDonald LR, Badawi RD (2011) Effect of object size on scatter fraction estimation methods for PET-a computer simulation study. IEEE Trans Nucl Sci 58:82-86

16. Konik A, Madsen MT, Sunderland JJ (2010) GATE simulations of human and small animal PET for determination of scatter fraction as a function of object size. IEEE Trans Nucl Sci 57:2558-2563

17. National Electrical Manufacturers Association (2008) NEMA Standards Publication NU 4-2008. Performance measurements of small animal positron emission tomographs. National Electrical Manufacturers Association, Rosslyn, VA

18. Wilson JM, Lokitz SJ, Turkington TG (2011) Development of a fillable, tapered PET/CT phantom. IEEE Transactions on Nuclear Science 58:651-659

19. Rominger A, Mille E, Zhang S, Boning G, Forster S, Nowak S et al (2010) Validation of the octamouse for simultaneous 18F-fallypride small-animal PET recordings from 8 mice. J Nucl Med 51:1576-1583

20. Jan S, Benoit D, Becheva E, Carlier T, Cassol F, Descourt P et al (2011) GATE V6: a major enhancement of the GATE simulation platform enabling modelling of CT and radiotherapy. Phys Med Biol 56:881-901

21. Agostinelli S, Allison J, Amako K, Apostolakis J, Araujo H, Arce P et al (2003) GEANT4 - a simulation toolkit. Nucl Instrum Meth A 506:250-303

22. Wong W-H, Li H, Xie S, Ramirez R, Kim S, Uribe J, et al (2003) Design of an inexpensive high-sensitivity rodent-research PET camera (RRPET). In: Li H (ed) IEEE Nuclear Science Symposium Conference Record, pp 2058-2062

23. Xie S, Ramirez R, Liu Y, Xing T, Uribe J, Li H et al (2005) A pentagon photomultiplier-quadrant-sharing BGO detector for a rodent research PET (RRPET). IEEE Trans Nucl Sci 52:210-216

24. Wong W-H, Li H, Uribe J, Baghaei H, Wang Y, Yokoyama S (2001) Feasibility of a high-speed gamma-camera design using the high-yieldpileup-event-recovery method. J Nucl Med 42:624-632

25. Zeraatkar N, Ay MR, Kamali-Asl AR, Zaidi H (2011) Accurate Monte Carlo modeling and performance assessment of the X-PET subsystem of the FLEX Triumph ${ }^{\mathrm{TM}}$ preclinical PET/CT scanner. Med Phys 38:1217-1225

26. Rechka S, Fontaine R, Rafecas M, Lecomte R (2009) Development and validation of a GATE simulation model for the LabPET scanner. IEEE Trans Nucl Sci 56:3672-3679

27. Daube-Witherspoon ME, Muehllehner G (1987) Treatment of axial data in three-dimensional PET. J Nucl Med 28:1717-1724

28. Bergeron M, Cadorette J, Beaudoin J-F, Tetrault M-A, Viscogliosi N, Selivanov V, et al. (2008) Imaging performance of the LabPET ${ }^{\mathrm{TM}}$ APDbased digital PET scanner. IEEE Nuclear Science Symposium Conference Record, pp 3841-3845.

29. Segars WP, Tsui BM, Frey EC, Johnson GA, Berr SS (2004) Development of a 4-D digital mouse phantom for molecular imaging research. Mol Imaging Biol 6:149-159

30. Zhang G, Xie T, Bosmans H, Liu Q (2009) Development of a rat computational phantom using boundary representation method for Monte Carlo simulation in radiological imaging. Proc IEEE 97:20062014

31. Wang Y, Seidel J, Tsui BM, Vaquero JJ, Pomper MG (2006) Performance evaluation of the GE Healthcare eXplore VISTA dualring small-animal PET scanner. J Nucl Med 47:1891-1900

32. Rechka S, Fontaine R, Lecomte R, Rafecas M (2009) LabPET intercrystal scatter study using GATE. IEEE Nuclear Science Symposium Conference Record (NSS/MIC), pp 3988-3994.

33. Chatziioannou AF, Qinan B, Karakatsanis N (2008) System sensitivity in preclinical small animal imaging. 5th IEEE International Symposium on Biomedical Imaging: From Nano to Macro, ISBI, pp 1417-1420.

34. Naidoo-Variawa S, Lehnert W, Banati RB, Meikle SR (2011) Scatter correction for large non-human primate brain imaging using microPET. Phys Med Biol 56:2131-2143 